\title{
Finite-index normal subgroups of crystallographic space groups
}

\author{
Miles A. Clemens ${ }^{1}$, Branton J. Campbell ${ }^{1}$, Stephen P. Humphries ${ }^{2}$, Harold T. Stokes ${ }^{1}$ \\ ${ }^{I}$ Department of Physics \& Astronomy, Brigham Young University, Provo, Utah, USA \\ ${ }^{2}$ Department of Mathematics, Brigham Young University, Provo, Utah, USA
}

\begin{abstract}
Symmetry-lowering phase transitions give rise to crystal domain patterns in ferroelectrics and many other types of materials. If the order parameter responsible for the transition possesses child space-group $H$, which is a subgroup of the parent space group $G$, the crystal domains of the child phase are associated with equivalent directions of this order-parameter, which are in one-toone correspondence with the set $S$ of cosets of $H$ in $G$. While $H$ is not normal in general, the normal core $N$ of $H$ in $G$ is a finite-index normal subgroup of $G$, for which the quotient group $G / N$ has a well-defined permutation action on $S$. In this sense, $G / N$ is the symmetry group of $S$, and can be used to classify its symmetry-inequivalent domain pairs [1-3]. The importance of finiteindex normal subgroups to the study of crystal-domain configurations motivates us to tabulate them for each crystallographic space group.
\end{abstract}

[1] D. M. Hatch, R. A. Hatt, H. T. Stokes, Ferroelectrics 191, 29-35 (1997).

[2] D. B. Litvin, Acta Cryst. A55, 884-890 (1999).

[3] J. Fuksa, Ferroelectrics 240, 1275-1284 (2000). 